\title{
Constant Accelerated Flow for a Third-Grade Fluid in a Porous Medium and a Rotating Frame with the Homotopy Analysis Method
}

\author{
Zainal Abdul Aziz, ${ }^{1}$ Mojtaba Nazari, ${ }^{1}$ \\ Faisal Salah, ${ }^{2}$ and Dennis Ling Chuan Ching $^{3}$ \\ ${ }^{1}$ Department of Mathematical Sciences, Faculty of Science, Universiti Teknologi Malaysia, \\ 81310 Johor Bahru, Malaysia \\ ${ }^{2}$ Department of Mathematics, Faculty of Science, University of Kordofan, North Kordofan State, \\ El-Obied 51111, Sudan \\ ${ }^{3}$ Department of Fundamental and Applied Sciences, Universiti Teknologi PETRONAS, \\ 31750 Tronoh, Malaysia
}

Correspondence should be addressed to Zainal Abdul Aziz, abdulazizzainal@gmail.com

Received 2 October 2012; Accepted 7 November 2012

Academic Editor: Yuji Liu

Copyright (C) 2012 Zainal Abdul Aziz et al. This is an open access article distributed under the Creative Commons Attribution License, which permits unrestricted use, distribution, and reproduction in any medium, provided the original work is properly cited.

The homotopy analysis method (HAM) is applied to obtain the approximate analytic solution of a constant accelerated flow for a third-grade fluid in a porous medium and a rotating frame. HAM is an analytic technique which provides us with a new way to obtain series solutions of such nonlinear problems. The approximate analytic solution for constant accelerated flow is obtained by using HAM. HAM contains the auxiliary parameter $\hbar$, which provides us with a straightforward way to obtain the convergence region of the series solution. Graphical results are plotted and the consequences discussed. The obtained solutions clearly satisfy the governing equations and all the imposed initial and boundary conditions. Many interesting results can be obtained as the special cases of the presented analysis. The influence of the material parameters of a third-grade fluid and rotation upon the velocity field is finally deliberated.

\section{Introduction}

It is difficult to solve nonlinear problems, especially by an analytic technique. The homotopy analysis method (HAM) [1, 2] is an analytic technique for nonlinear problems, which was initially introduced by Liao in 1992. This method has been successfully applied to many nonlinear problems in engineering and science, such as the magnetohydrodynamic flows of non-Newtonian fluids over a stretching sheet [3], the boundary-layer flows over an impermeable stretched plate [4], the nonlinear model of the combined convective and 
radiative cooling of a spherical body [5], the exponentially decaying boundary layers [6], and the unsteady boundary layer flows over a stretching flat plate [7]. Thus, the validity, effectiveness, and flexibility of the HAM are verified via all of the successful applications. Also, many types of nonlinear problems were solved with HAM by others [8-22]. The equations governing the flow of a viscous fluid, namely, the Navier-Stokes equations, are nonlinear. But there are several complicated fluids which are not well described by these equations. Due to this reason, many constitutive equations have been proposed for the nonNewtonian fluids. The equations for non-Newtonian fluids are much complicated and of higher order than the Navier-Stokes equations. Even the various investigators are presently engaged in finding the solutions for such flow problems. Some recent attempts relevant to the flows of non-Newtonian fluids in nonrotating frame are given in [23-30]. The study of rotating flows has gained considerable importance due to their applications in cosmical and geophysical fluid dynamics. Recently, there are a few works in this area such as an oscillating hydromagnetic non-Newtonian flow in a rotating system [31], a hydromagnetic Couette flow of an Oldroyd-B fluid in a rotating system [32], and Stokes' first problem for the rotating flow of a third-grade fluid [33]. In all of these above-mentioned studies, the rotating flows of non-Newtonian fluids have been studied as a boundary value problem. Therefore, all the mentioned studies lack the features of unsteadiness. This study fills the gap in this area. Thus, the main objective of the present study is to obtain an approximate analytic solution for unsteady third-grade fluid in a rotating frame. The flow in the fluid is induced by a constant accelerated plate. In addition the graphical results are plotted and discussed, where the effect of the material parameters of third-grade fluid and rotation upon the velocity field is deliberated.

\section{Governing Equations}

Consider an incompressible third-grade fluid occupying the space $z>0$. The plate at $z=0$ is moved with a constant acceleration $A$ in the $x$-direction for $t>0$ and induced the motion in the fluid. Both the fluid and plate are in a solid body rotation. Initially the fluid and plate are at rest. The laws which govern the flow are [33]

$$
\begin{gathered}
\operatorname{div} \mathbf{V}=0 \\
\rho\left[\frac{\partial \mathbf{V}}{\partial t}+(\mathbf{V} \cdot \nabla) \mathbf{V}+2 \boldsymbol{\Omega} \times \mathbf{V}+\boldsymbol{\Omega} \times(\boldsymbol{\Omega} \times r)\right]=-\nabla p+\operatorname{div} \mathbf{T}
\end{gathered}
$$

in which $\mathbf{V}$ is the velocity, $\rho$ the fluid density, $t$ the time, $p$ the hydrostatic pressure, $\mathbf{T}$ the extra stress tensor, $\boldsymbol{\Omega}$ the constant angular velocity, and $r$ the radial coordinate with $r^{2}=x^{2}+y^{2}$.

The extra stress tensor $\mathbf{T}$ in a third-grade fluid is [33]

$$
\mathbf{T}=\mu A_{1}+\alpha_{1} A_{2}+\alpha_{2} A_{1}^{2}+\beta_{1} A_{3}+\beta_{2}\left(A_{2} A_{1}+A_{1} A_{2}\right)+\beta_{3}\left(\operatorname{tr} A_{1}^{2}\right) A_{1} .
$$

Here $\mu$ is the dynamic viscosity; $\alpha_{i}(i=1,2)$ and $\beta_{j}(j=1,2,3)$ are the material constants. The kinematical tensors $A_{n}$ are

$$
\begin{gathered}
A_{1}=(\operatorname{grad} \mathbf{V})+(\operatorname{grad} \mathbf{V})^{T} \\
A_{n+1}=\left(\frac{\partial}{\partial t}+(\mathbf{V} \cdot \nabla)\right) A_{n}+A_{n}(\operatorname{grad} \mathbf{V})+(\operatorname{grad} \mathbf{V})^{T} A_{n}, \quad n>1 .
\end{gathered}
$$


The thermodynamics of the fluid requires that [34]

$$
\mu \geq 0, \quad \alpha_{1} \geq 0, \quad\left|\alpha_{1}+\alpha_{2}\right| \leq \sqrt{24 \mu \beta_{3}}, \quad \beta_{1}=\beta_{1}=0, \quad \beta_{3} \geq 0 .
$$

Therefore, (2.3) can be written as

$$
\mathbf{T}=\left[\mu+\beta_{3}\left(\operatorname{tr} A_{1}^{2}\right)\right] A_{1}+\alpha_{1} A_{2}+\alpha_{2} A_{1}^{2}
$$

Since the plate is infinite, so the velocity field $V$ for the present flow is

$$
\mathbf{V}=[u(z, t), v(z, t), w(z, t)]
$$

which together with the incompressibility condition yields $w=0(u, v$, and $w$ are the velocities in the $x, y, z$ directions, resp.).

Substituting (2.6) and (2.7) into (2.2), one obtains

$$
\begin{gathered}
\frac{\partial u}{\partial t}-2 \Omega v=-\frac{1}{\rho} \frac{\partial \hat{p}}{\partial x}+\frac{1}{\rho}\left[\mu \frac{\partial^{2} u}{\partial z^{2}}+\alpha_{1} \frac{\partial^{3} u}{\partial z^{2} \partial t}+2 \beta_{3} \frac{\partial}{\partial z}\left(\frac{\partial u}{\partial z}\left\{\left(\frac{\partial u}{\partial z}\right)^{2}+\left(\frac{\partial v}{\partial z}\right)^{2}\right\}\right)\right] \\
\frac{\partial v}{\partial t}+2 \Omega u=-\frac{1}{\rho} \frac{\partial \hat{p}}{\partial y}+\frac{1}{\rho}\left[\mu \frac{\partial^{2} v}{\partial z^{2}}+\alpha_{1} \frac{\partial^{3} v}{\partial z^{2} \partial t}+2 \beta_{3} \frac{\partial}{\partial z}\left(\frac{\partial v}{\partial z}\left\{\left(\frac{\partial u}{\partial z}\right)^{2}+\left(\frac{\partial v}{\partial z}\right)^{2}\right\}\right)\right] \\
0=-\frac{1}{\rho} \frac{\partial \hat{p}}{\partial z}
\end{gathered}
$$

where the modified pressure

$$
\widehat{p}=p-\frac{\rho}{2} \Omega^{2}\left(x^{2}+y^{2}\right)
$$

and (2.10) shows that $\hat{p} \neq \hat{p}(z)$.

The boundary and initial conditions corresponding to constant accelerated plate are

$$
\begin{aligned}
& u=A t, \quad v=0 \quad \text { at } z=0, t>0, \\
& u \longrightarrow 0, \quad v \longrightarrow 0 \quad \text { as } z \longrightarrow \infty \forall t \\
& u(z, 0)=0, \quad v(z, 0)=0, \quad z>0
\end{aligned}
$$

where $A$ is constant accelerated.

Combining (2.8) and (2.9) and then neglecting the pressure gradient, we have

$$
\frac{\partial F}{\partial t}+2 i \Omega F=v \frac{\partial^{2} F}{\partial z^{2}}+\frac{\alpha_{1}}{\rho} \frac{\partial^{3} F}{\partial z^{2} \partial t}+\frac{2 \beta_{3}}{\rho} \frac{\partial}{\partial z}\left\{\left(\frac{\partial F}{\partial z}\right)^{2}\left(\frac{\partial \bar{F}}{\partial z}\right)\right\},
$$


in which $v$ is the kinematic viscosity and

$$
F=u+i v, \quad \bar{F}=u-i v .
$$

The boundary and initial conditions now are

$$
F(0, t)=A t, \quad F(z, t) \longrightarrow 0 \quad \text { as } z \longrightarrow \infty, \quad F(z, 0)=0 .
$$

The above equation can be normalized using the following dimensionless parameters:

$$
f=\frac{F}{(v A)^{1 / 3}}, \quad \eta=z\left(\frac{A}{v^{2}}\right)^{1 / 3}, \quad \tau=t\left(\frac{A^{2}}{v}\right)^{1 / 3}, \quad \Omega\left(\frac{v}{A^{2}}\right)^{1 / 3}=\Omega_{1}
$$

Accordingly, the above equations, after dropping the asterisks, take the form

$$
\begin{aligned}
& \frac{\partial f}{\partial \tau}+2 i \Omega_{1} f=\frac{\partial^{2} f}{\partial \eta^{2}}+a \frac{\partial^{3} f}{\partial \eta^{2} \partial \tau}+2 b \frac{\partial}{\partial \eta}\left\{\left(\frac{\partial f}{\partial \eta}\right)^{2}\left(\frac{\partial \bar{f}}{\partial \eta}\right)\right\}, \\
& f(0, \tau)=\tau, \quad f(\eta, \tau) \longrightarrow 0 \quad \text { as } \eta \longrightarrow \infty, \quad f(\eta, 0)=0,
\end{aligned}
$$

in which

$$
a=\frac{\alpha_{1}}{\rho}\left(\frac{A^{2}}{v^{4}}\right)^{1 / 3}, \quad b=\frac{\beta_{3}}{\rho}\left(\frac{A^{4}}{v^{5}}\right)^{1 / 3}
$$

\section{Essential Ideas Related to the Homotopy Analysis Method (HAM)}

Consider a nonlinear equation in a general form:

$$
\mathcal{N}[u(r, t)]=0,
$$

where $\mathcal{N}$ is a nonlinear operator and $u(r, t)$ is unknown function. Let $u_{0}(r, t)$ denote an initial guess of the exact solution $u(r, t), \hbar \neq 0$ an auxiliary parameter, $\mathscr{H}(r, t) \neq 0$ an auxiliary function, and $\mathcal{L}$ an auxiliary linear operator, $Q \in[0,1]$ as an embedding parameter, and by means of homotopy analysis method, we construct the so-called zeroth-order deformation equation

$$
(1-Q) \mathcal{L}\left[\phi(r, t ; Q)-u_{0}(r, t)\right]=Q \hbar \mathscr{\ell}(r, t) \mathcal{N}[\phi(r, t ; Q)]
$$

It is very significant that one has great freedom to choose auxiliary objects in HAM in accordance to the rule of its solution expression. 
In many cases, by means of analyzing its physical background, its initial/boundary conditions, and/or its type of nonlinearity, we might know what kinds of base functions are proper to represent the solution, even without solving a given nonlinear problem. Furthermore, it is important to obey the rule of solution expression denoted by Liao [1], and thus the auxiliary function $H(r, t)$ should be chosen so that the particular solution of the high-order deformation equations (e.g., (3.8)) must be expressed by a sum of the base functions. Note that we have established the initial and base functions founded on boundary conditions.

Clearly, when $Q=0,1$ it holds

$$
\phi(r, t ; 0)=u_{0}(r, t), \quad \phi(r, t ; 1)=u(r, t),
$$

respectively. Then as long as $Q$ increases from 0 to 1 , the solution $\phi(r, t ; Q)$ varies from the initial guess $u_{0}(r, t)$ to the exact solution $u(r, t)$. Liao [2] by the Taylor theorem expanded $\phi(r, t ; Q)$ in a power series of $Q$ as follows:

$$
\phi(r, t ; \mathcal{Q})=\phi(r, t ; 0)+\sum_{m=1}^{\infty} u_{m}(r, t) Q^{m}
$$

where

$$
u_{m}(r, t)=\left.\frac{1}{m !} \frac{\partial^{m} \phi(r, t ; Q)}{\partial Q^{m}}\right|_{Q=0}
$$

The convergence of the series (3.4) depends upon the auxiliary parameter $\hbar$, auxiliary function $\mathscr{\ell}(r, t)$, initial guess $u_{0}(r, t)$, and auxiliary linear operator $\mathcal{L}$. If they are chosen properly, the series (3.4) is convergent at $Q=1$, one has

$$
u(r, t)=u_{0}(r, t)+\sum_{m=1}^{\infty} u_{m}(r, t)
$$

According to definition (3.5), the governing equation can be inferred from the zeroth-order deformation equation (3.2). We define the vector

$$
\overrightarrow{u_{n}(r, t)}=\left\{u_{0}(r, t), u_{1}(r, t), \ldots, u_{n}(r, t)\right\}
$$


Differentiating the zeroth-order deformation equation (3.2) $m$-times with respect to $Q$, dividing them by $m$ !, and finally setting $Q=0$, we obtain the so-called $m$ th-order deformation equation:

$$
\mathcal{L}\left[u_{m}(r, t)-\chi_{m} u_{m-1}(r, t)\right]=\hbar \mathscr{\ell}(r, t) \mathcal{R}_{m}\left(u_{m-1}, r, t\right),
$$

where

$$
\begin{gathered}
X_{m}= \begin{cases}0, & m \leq 1, \\
1, & m>1,\end{cases} \\
\mathcal{R}_{m}\left(u_{m-1}, r, t\right)=\left.\frac{1}{(m-1) !}\left\{\frac{\partial^{m-1}}{\partial Q^{m-1}} N\left[\sum_{m=0}^{\infty} u_{m}(r, t) Q^{m}\right]\right\}\right|_{Q=0} .
\end{gathered}
$$

Theorem 3.1 (Liao [2]). As long as the series (3.6) is convergent, it is convergent to the exact solution of (3.1).

Note that homotopy analysis method contains the auxiliary parameter $\hbar$, which provides us with the control and adjustment for the convergence of the series solution (3.6).

\section{HAM Solution}

For HAM solution of (2.17), we choose

$$
f_{0}(\eta, \tau)=\tau e^{-\eta}
$$

as the initial guess and

$$
\mathcal{L}[f(\eta, \tau ; Q)]=\frac{\partial^{2} f(\eta, \tau ; Q)}{\partial \eta^{2}}+\frac{\partial f(\eta, \tau ; Q)}{\partial \eta}
$$

as the auxiliary linear operator satisfying

$$
\mathcal{L}\left[c_{1} \tau+c_{2} \tau e^{-\eta}\right]=0 .
$$




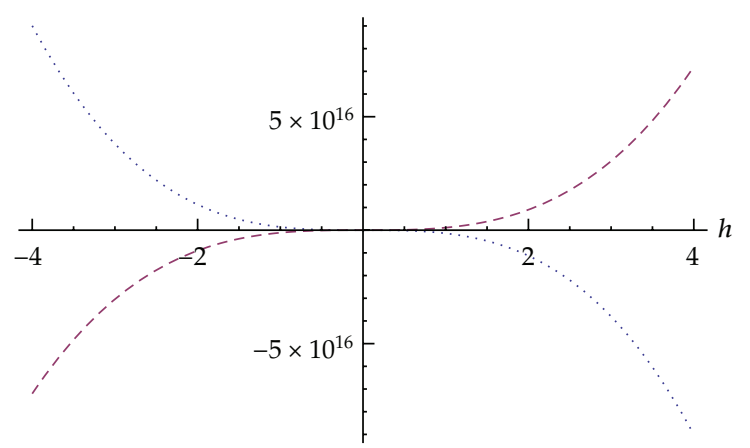

(a)

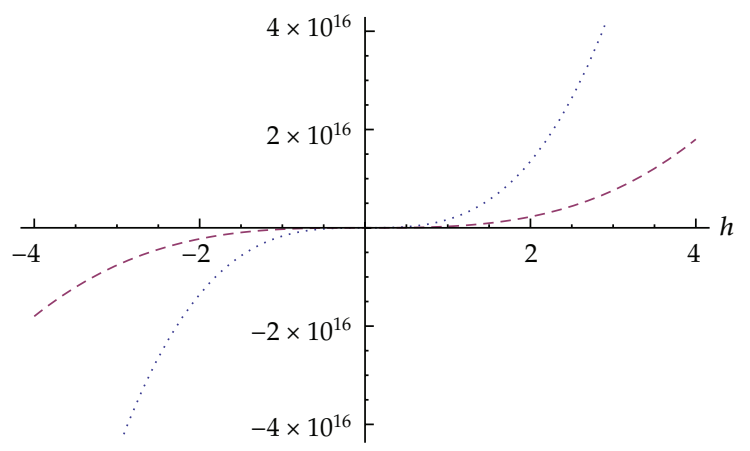

(b)

Figure 1: The $\hbar$-curve at 4 th-order approximation with tiny dashes: $f(0.1,0.1)$ with large dashes: $\dot{f}(0.1,0.1)$.

We consider the auxiliary function

$$
\mathscr{H}(r, t)=1
$$

a zeroth-order deformation problem

$$
\begin{gathered}
(1-Q) \mathcal{L}\left[f(\eta, \tau ; Q)-f_{0}(\eta, \tau ; Q)\right]=Q \hbar \mathcal{N}[f(\eta, \tau ; Q)], \\
f_{0}(\eta, \tau)=\tau e^{-\eta}, \\
\mathcal{N}[f(\eta, \tau ; Q)]=\frac{\partial f(\eta, \tau ; Q)}{\partial \tau}+2 i \Omega_{1} f(\eta, \tau ; Q)-\frac{\partial^{2} f(\eta, \tau ; Q)}{\partial \eta^{2}}-a \frac{\partial^{3} f(\eta, \tau ; Q)}{\partial \eta^{2} \partial \tau} \\
-2 b \frac{\partial}{\partial \eta}\left\{\left(\frac{\partial f(\eta, \tau ; Q)}{\partial \eta}\right)^{2}\left(\frac{\partial f^{\star}(\eta, \tau ; Q)}{\partial \eta}\right)\right\},
\end{gathered}
$$




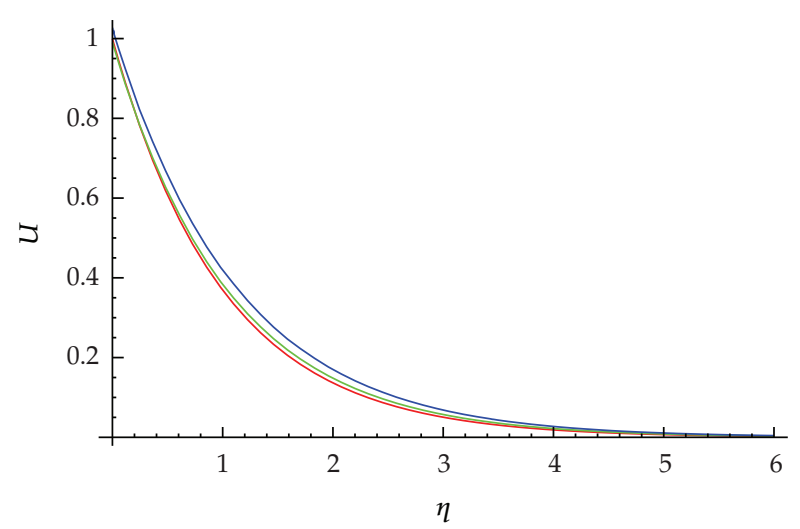

(a)

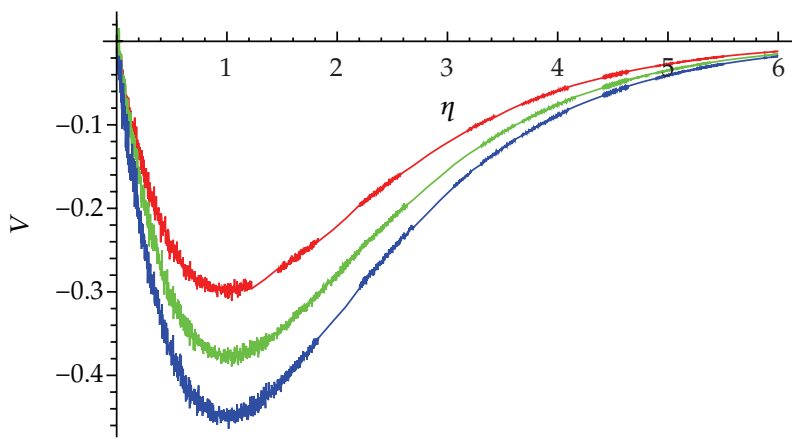

(b)

Figure 2: Influence of the third grade parameter on the velocity distribution for $\tau=1 ; a=0.1 ; \Omega_{1}=1$ : red color $b=0.001$, green color $b=0.002$, blue color $b=0.003$.

in which

$$
f^{\star}(\eta, \tau ; Q)=\bar{f}(\eta, \tau ; Q)
$$

The $m$ th-order deformation problem is given by

$$
\begin{gathered}
\mathcal{L}\left[f_{m}(\eta, \tau)-x_{m} f_{m-1}(\eta, \tau)\right]=\hbar\left[\frac{\partial f_{m-1}}{\partial \tau}-f_{m-1}^{\prime \prime}-a \frac{\partial f_{m-1}^{\prime \prime}}{\partial \tau}+2 i \Omega_{1} f_{m-1}\right. \\
\left.-2 b \sum_{n=0}^{m-1} f_{m-1-n}^{\prime} \sum_{i=0}^{n}\left\{f_{n-i}^{\prime}\left(f_{i}^{\prime \prime}\right)^{\star}+2 f_{n-i}^{\prime \prime}\left(f_{i}^{\prime}\right)^{\star}\right\}\right] \\
f_{m}(0, \tau)=0, \quad f_{m}(\infty, \tau)=0, \quad f_{m}(\eta, 0)=0, \quad(m \geq 1) .
\end{gathered}
$$




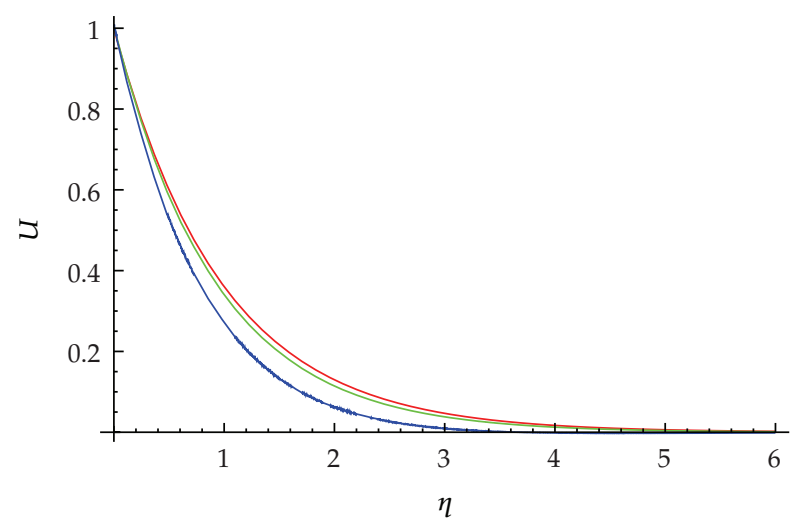

(a)

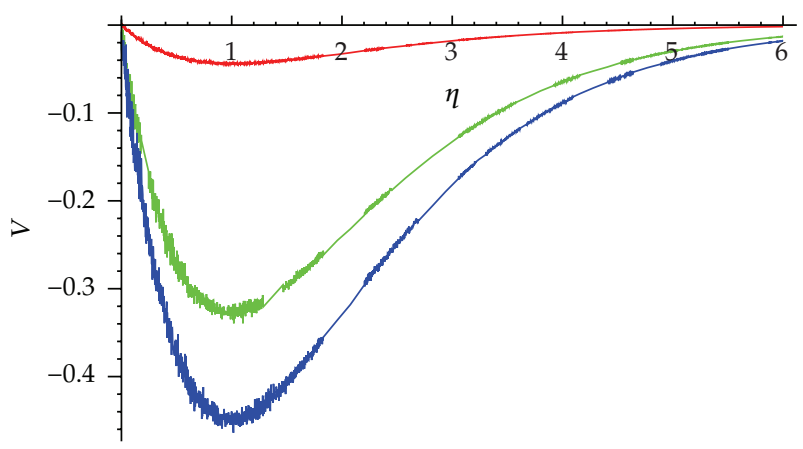

(b)

Figure 3: The influence of the angular velocity on the velocity distribution for $\tau=1 ; a=0.1 ; b=0.001$; red color $\Omega_{1}=1$; green color $\Omega_{1}=0.5$, blue color $\Omega_{1}=1$.

We can use MATHEMATICA for solving the set of linear equations (4.7) with condition (4.8). It is found that the solution in a series form is given by

$$
\begin{aligned}
f(\eta, \tau)=(\tau) e^{-\eta}+e^{-5 \eta}( & 5.9216 \times 10^{9} e^{4 \eta} \hbar-5.9216 \times 10^{9} e^{4 \eta} \hbar-5.9216 \\
& \times 10^{9} e^{4 \eta} \hbar \tau+5.9216 \times 10^{9} e^{4 \eta} \hbar \tau-5.9216 \\
& \times 10^{9} e^{4 \eta} \hbar a+5.9216 \times 10^{9} e^{4 \eta} \hbar a-e^{2 \eta} \hbar \tau^{3} b \\
& +e^{4 \eta} \hbar \tau^{3} b+\left(1.18432 \times 10^{10} i\right) e^{4 \eta} \hbar \tau \Omega_{1} \\
& \left.-\left(1.18432 \times 10^{10} i\right) e^{4 \eta} \hbar \tau \Omega_{1}\right)+\cdots
\end{aligned}
$$

The analytic solution given by (4.9) contains the auxiliary parameter $\hbar$, which influences the convergence region and the rate of approximation for the HAM solution. In Figures 1(a) and $1(\mathrm{~b})$, the $\hbar$-curves are plotted for $f(\eta, \tau), \dot{f}(\eta, \tau)$ when $\eta=\tau=0.1, a=0.1, b=0$, and $\Omega_{1}=1$ at 4th-order approximation for real and imaginary part of $f(\eta, \tau)$, respectively. 


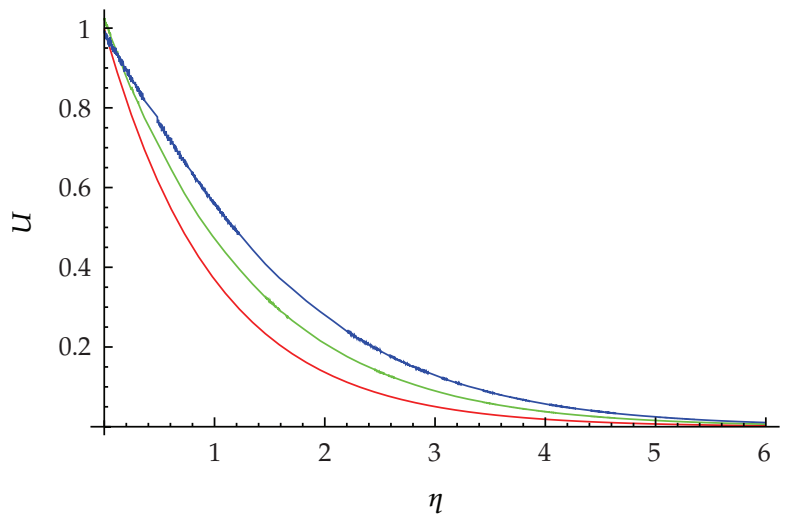

(a)

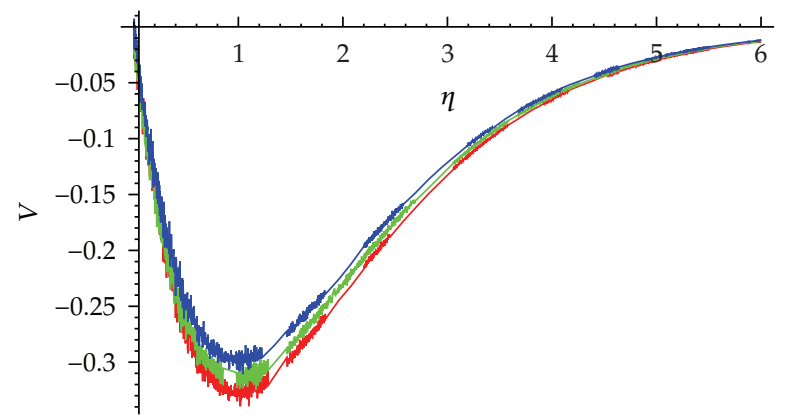

(b)

Figure 4: Influence of the various values of the second-grade parameter on the velocity distribution for $\tau=1 . b=0 ; \Omega_{1}=1$ : red color $a=0.1$, green color $a=0.5$, blue color $a=1$.

As pointed out by Liao, the valid region of $\hbar$ is a horizontal line segment on the $\hbar$-curve graph, and this is obviously shown in Figures 1(a) and 1(b). It is clear that the valid region for this case is $-1<\hbar<0.5$; that is, both Figures $1(\mathrm{a})$ and $1(\mathrm{~b})$ indicate that the convergence of the HAM solution is valid for values of $\hbar$ between -1 and 0.5 . In this case for $\hbar=-0.1$, the obtained results are summarized in Figures 2-6.

\section{HAM Results and Discussions}

The aim of this section is to address the influence of several pertinent parameters on the dimensionless velocity field components. In this paper, the homotopy analysis method (HAM) [2] is applied to obtain the solution of the nonlinear differential equation (2.17) with conditions (2.18). HAM provides us with a convenient way to control the convergence of the approximation series, which is a fundamental qualitative difference in analysis between HAM and other methods. Solutions for the non-Newtonian fluid models are obtained for some values of $\tau$. The HAM solution $f$ is used to express the nondimensional velocity profile. Graphical results for the flow are obtained for various values of the parameters $a, b, \Omega_{1}$, and $\tau$. The insets (a) and (b) in each plot represent the real and imaginary parts of the derived velocity profile, respectively. 


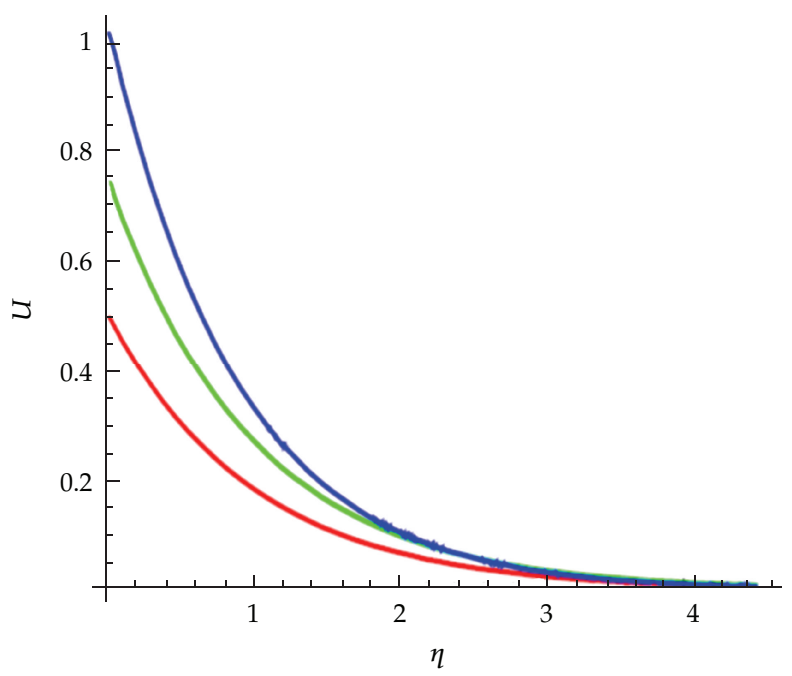

(a)

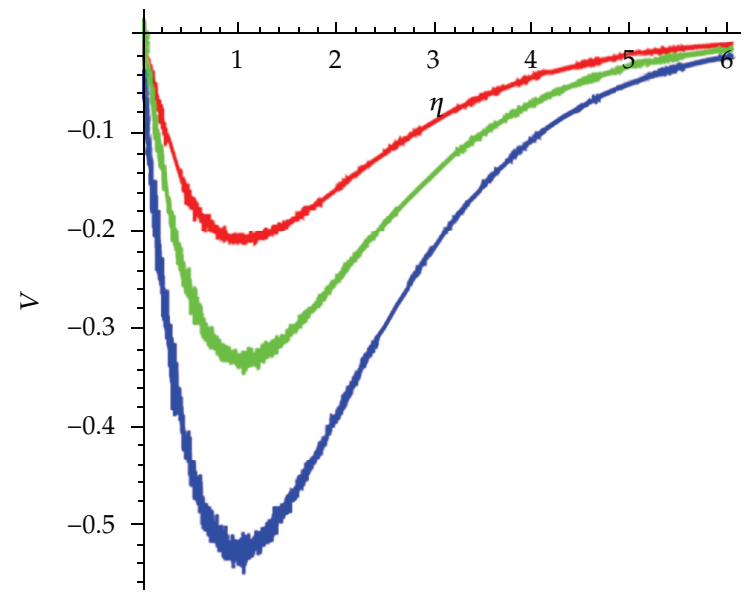

(b)

Figure 5: Influence of various values of $\tau$ on the velocity distribution. $a=0.1 ; b=0.005 ; \Omega_{1}=1$ : red color $\tau=0.5$, green color $\tau=0.75$, blue color $\tau=1$.

Figures 2(a) and 2(b) present the velocity profile $f$ for various values of the material constant, third-grade parameter $b$. These figures indicate that increasing the parameter $b$ would increase the real part of the velocity profile, whiles the imaginary part of the velocity profile decreases for large values of $b$. Figures 3(a) and 3(b) show the influence of the angular velocity, that is, the rotational parameter $\Omega_{1}$ on the velocity profile $f$. It is clear from the figures that the increase in $\Omega_{1}$ results in the decrease in the real and imaginary parts of the velocity profile. In Figures 4(a) and 4(b), it is noted that the velocity profile increases in the real part and the imaginary part by increasing the second-grade parameter $a$. Figures $5(a)$ and 5 (b) show how the velocity profile changes for various values of time $\tau$. It is found that here the real part of the velocity profile increases whereas the imaginary part of the velocity profile decreases by increasing $\tau$. In Figures 6(a) and 6(b), the velocity distribution is presented in 


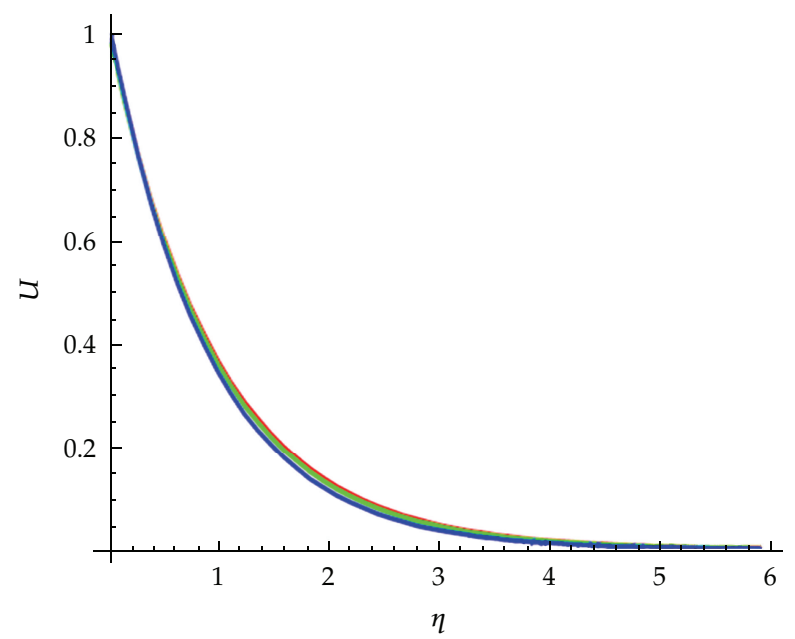

(a)

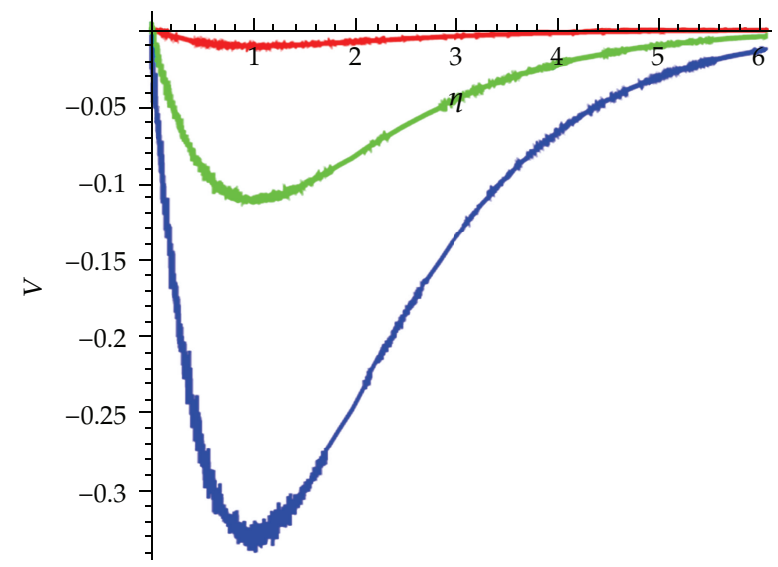

(b)

Figure 6: Influence of the angular velocity on the velocity distribution for the Newtonian case for $\tau=1$; $a=0 ; b=0$ : red color $\Omega_{1}=0.1$, green color $\Omega_{1}=0.5$, blue color $\Omega_{1}=1$.

the Newtonian case $(a=b=0)$ for the various values of $\Omega_{1}$. It is observed that the effect of $\Omega_{1}$ in a Newtonian fluid and a third-grade fluid is similar.

\section{Concluding Remarks}

In this paper, the unsteady rotating flow engendered by a constant accelerated plate has been studied via the use of the homotopy analysis method. From the presented analysis, results for the real and imaginary parts of the velocity field are presented. It is observed that at $\tau=1$ and different values of $\Omega_{1}$, the flow characteristics in a third-grade fluid are similar to that of Newtonian fluid.

Thus, these examples show the flexibility and potential of the homotopy analysis method for solving complicated nonlinear problems in engineering. 


\section{Acknowledgments}

This research is partially funded by the MOHE FRGS Vote no. 78675 and the UTM RUG Vote no. 05J13. M. Nazari is thankful to the UTM for the International Doctoral Fellowship (IDF).

\section{References}

[1] S. J. Liao, The proposed homotopy analysis technique for the solution of nonlinear problems [Ph.D. thesis], Jiao University, 1992.

[2] S. Liao, Ed., Beyond Perturbation: Introduction to the Homotopy Analysis Methodn, vol. 2, Chapman and Hall, Boca Raton, Fla, USA, 2004

[3] S.-J. Liao, "On the analytic solution of magnetohydrodynamic flows of non-Newtonian fluids over a stretching sheet," Journal of Fluid Mechanics, vol. 488, pp. 189-212, 2003.

[4] S. Liao, "A new branch of solutions of boundary-layer flows over an impermeable stretched plate," International Journal of Heat and Mass Transfer, vol. 48, no. 12, pp. 2529-2539, 2005.

[5] S. Liao, J. Su, and A. T. Chwang, "Series solutions for a nonlinear model of combined convective and radiative cooling of a spherical body," International Journal of Heat and Mass Transfer, vol. 49, no. 15-16, pp. 2437-2445, 2006.

[6] S. Liao and E. Magyari, "Exponentially decaying boundary layers as limiting cases of families of algebraically decaying ones," Zeitschrift für Angewandte Mathematik und Physik, vol. 57, no. 5, pp. 777792, 2006.

[7] S. Liao, "Series solutions of unsteady boundary-layer flows over a stretching flat plate," Studies in Applied Mathematics, vol. 117, no. 3, pp. 239-263, 2006.

[8] S. Abbasbandy, "The application of homotopy analysis method to nonlinear equations arising in heat transfer," Physics Letters A, vol. 360, no. 1, pp. 109-113, 2006.

[9] S. Abbasbandy, "The application of homotopy analysis method to solve a generalized Hirota-Satsuma coupled KdV equation," Physics Letters A, vol. 361, no. 6, pp. 478-483, 2007.

[10] S. Abbasbandy, "Homotopy analysis method for heat radiation equations," International Communications in Heat and Mass Transfer, vol. 34, no. 3, pp. 380-387, 2007.

[11] S. Abbasbandy and A. Shirzadi, "A new application of the homotopy analysis method: solving the Sturm-Liouville problems," Communications in Nonlinear Science and Numerical Simulation, vol. 16, no. 1, pp. 112-126, 2011.

[12] S. Abbasbandy and A. Shirzadi, "Homotopy analysis method for multiple solutions of the fractional Sturm-Liouville problems," Numerical Algorithms, vol. 54, no. 4, pp. 521-532, 2010.

[13] S. Abbasbandy and A. Shirzadi, "A new application of the homotopy analysis method: solving the Sturm-Liouville problems," Communications in Nonlinear Science and Numerical Simulation, vol. 16, no. 1, pp. 112-126, 2011.

[14] S. Abbasbandy and A. Shirzadi, "The series solution of problems in the calculus of variations via the homotopy analysis method," Zeitschrift fur Naturforschung A, vol. 64, no. 1-2, pp. 30-36, 2009.

[15] S. Abbasbandy and T. Hayat, "Solution of the MHD Falkner-Skan flow by homotopy analysis method," Communications in Nonlinear Science and Numerical Simulation, vol. 14, no. 9-10, pp. 35913598, 2009.

[16] M. Ayub, A. Rasheed, and T. Hayat, "Exact flow of a third grade fluid past a porous plate using homotopy analysis method," International Journal of Engineering Science, vol. 41, no. 18, pp. 2091-2103, 2003.

[17] T. Hayat and M. Khan, "Homotopy solutions for a generalized second-grade fluid past a porous plate," Nonlinear Dynamics, vol. 42, no. 4, pp. 395-405, 2005.

[18] T. Hayat, M. Khan, and M. Ayub, “On non-linear flows with slip boundary condition," Zeitschrift für Angewandte Mathematik und Physik, vol. 56, no. 6, pp. 1012-1029, 2005.

[19] S. Asghar, M. M. Gulzar, and T. Hayat, "Rotating flow of a third grade fluid by homotopy analysis method," Applied Mathematics and Computation, vol. 165, no. 1, pp. 213-221, 2005.

[20] M. Sajid, T. Hayat, and S. Asghar, "On the analytic solution of the steady flow of a fourth grade fluid," Physics Letters A, vol. 355, no. 1, pp. 18-26, 2006.

[21] Y. Tan and S. Abbasbandy, "Homotopy analysis method for quadratic riccati differntial equation," Communications in Nonlinear Science and Numerical Simulation, vol. 13, no. 3, pp. 539-546, 2008. 
[22] C. Wang, Y. Y. Wu, and W. Wu, "Solving the nonlinear periodic wave problems with the homotopy analysis method," Wave Motion, vol. 41, no. 4, pp. 329-337, 2005.

[23] C. Fetecau and C. Fetecau, "A new exact solution for the flow of a Maxwell fluid past an infinite plate," International Journal of Non-Linear Mechanics, vol. 38, no. 3, pp. 423-427, 2003.

[24] C. Fetecau and C. Fetecau, "The first problem of stokes for an Oldroyd-B fluid," International Journal of Non-Linear Mechanics, vol. 38, no. 10, pp. 1539-1544, 2003.

[25] C. Fetecau and C. Fetecau, "Starting solutions for some unsteady unidirectional flows of a second grade fluid," International Journal of Engineering Science, vol. 43, no. 10, pp. 781-789, 2005.

[26] C. Fetecau, T. Hayat, and C. Fetecau, "Steady-state solutions for some simple flows of generalized Burgers fluids," International Journal of Non-Linear Mechanics, vol. 41, no. 8, pp. 880-887, 2006.

[27] C. Fetecau and C. Fetecau, "On some axial Couette flows of non-Newtonian fluids," Zeitschrift für Angewandte Mathematik und Physik, vol. 56, no. 6, pp. 1098-1106, 2005.

[28] R. S. Reddy and M. Kumari, "Nonsimilar solutions for mixed convection in non-Newtonian fluids along a vertical plate in a porous medium," Transport in Porous Media, vol. 33, no. 1-3, pp. 295-307, 1998.

[29] W. Tan and T. Masuoka, "Stokes' first problem for a second grade fluid in a porous half-space with heated boundary," International Journal of Non-Linear Mechanics, vol. 40, no. 4, pp. 515-522, 2005.

[30] W. Tan and T. Masuoka, "Stokes' first problem for an Oldroyd-B fluid in a porous half space," Physics of Fluids, vol. 17, no. 2, Article ID 023101, 7 pages, 2005.

[31] T. Hayat, S. Nadeem, A. M. Siddiqui, and S. Asghar, "An oscillating hydromagnetic non-Newtonian flow in a rotating system," Applied Mathematics Letters, vol. 17, no. 5, pp. 609-614, 2004.

[32] T. Hayat, S. Nadeem, and S. Asghar, "Hydromagnetic couette flow of an Oldroyd-B fluid in a rotating system," International Journal of Engineering Science, vol. 42, no. 1, pp. 65-78, 2004.

[33] F. Shahzad, T. Hayat, and M. Ayub, "Stokes' first problem for the rotating flow of a third grade fluid," Nonlinear Analysis, vol. 9, no. 4, pp. 1794-1799, 2008.

[34] R. L. Fosdick and K. R. Rajagopal, "Thermodynamics and stability of fluids of third grade," Proceedings of the Royal Society of London A, vol. 369, no. 1738, pp. 351-377, 1980. 


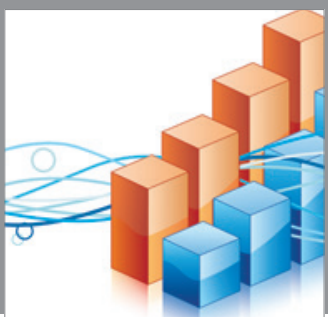

Advances in

Operations Research

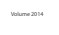

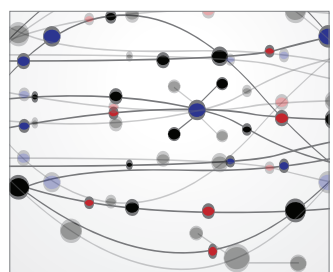

\section{The Scientific} World Journal
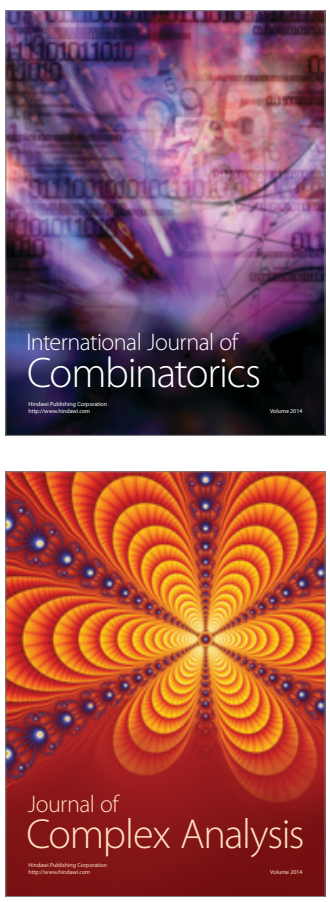

International Journal of

Mathematics and

Mathematical

Sciences
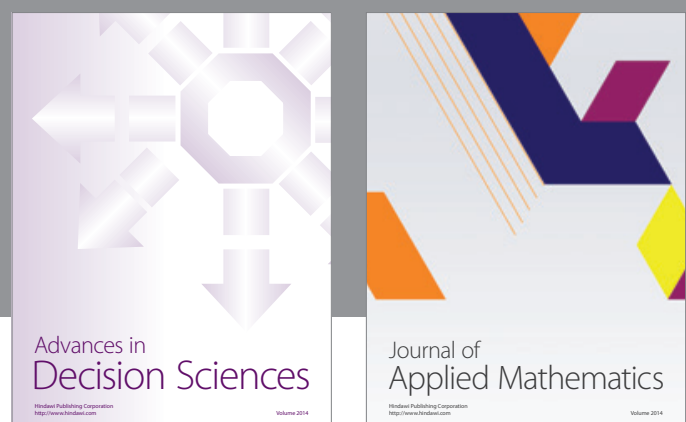

Journal of

Applied Mathematics
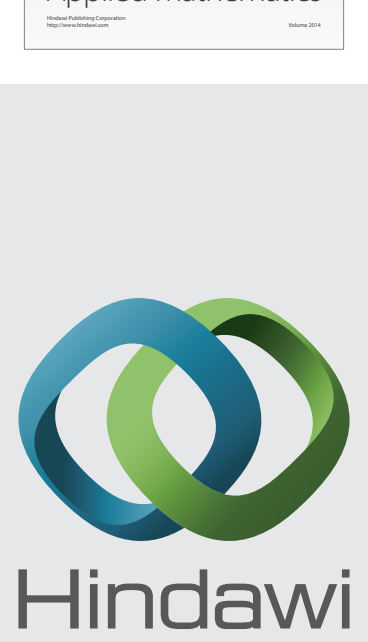

Submit your manuscripts at http://www.hindawi.com
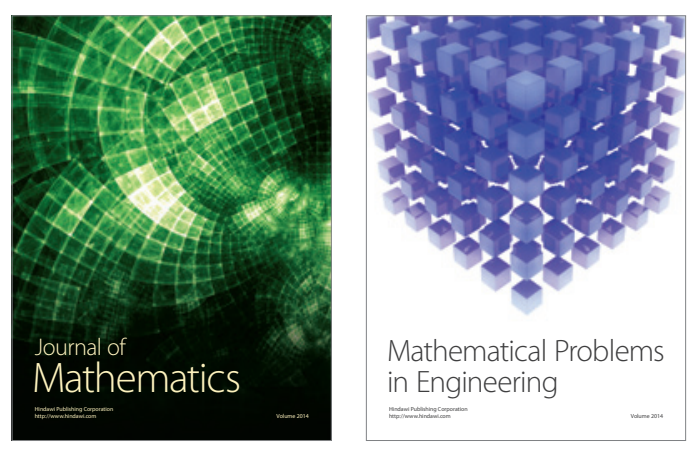

Mathematical Problems in Engineering
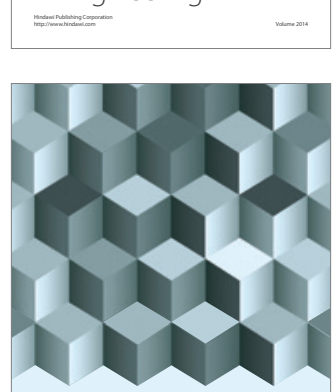

Journal of

Function Spaces
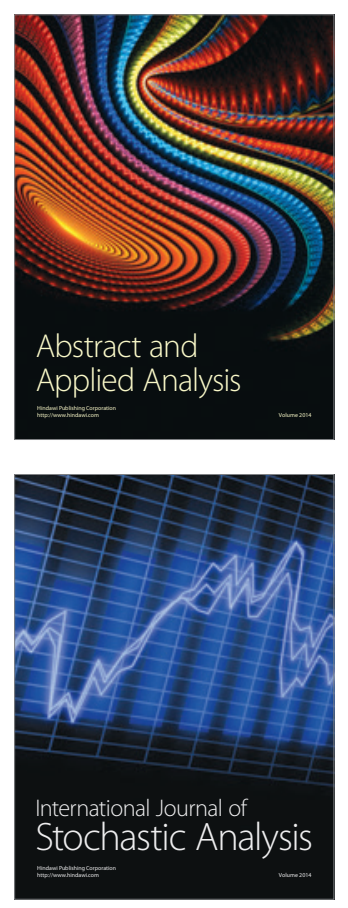

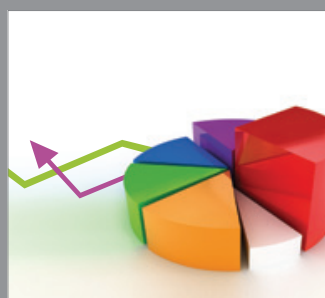

ournal of

Probability and Statistics

Promensencen
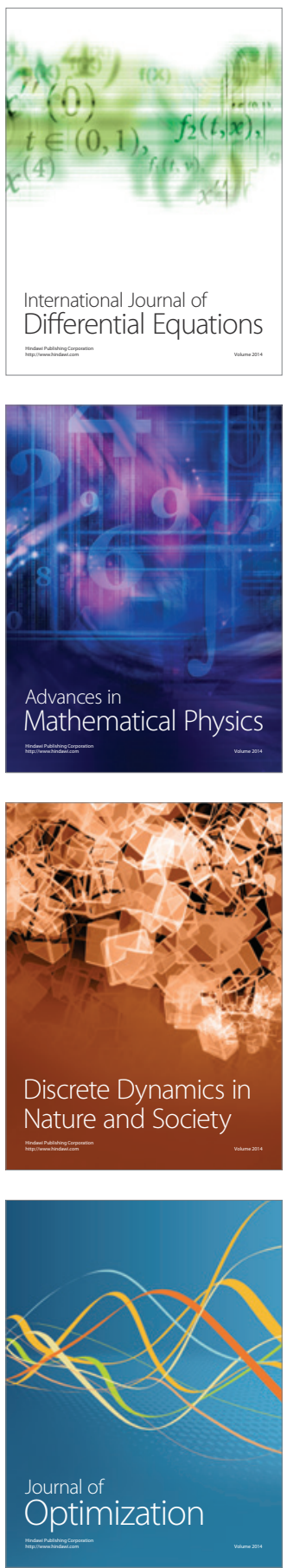